\title{
RESEARCH
}

Open Access

\section{Postnatal growth of preterm infants during the first two years of life: catch-up growth accompanied by risk of overweight}

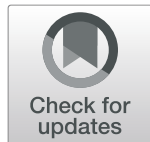

Junyan Han ${ }^{1,2+}$, Yuan Jiang ${ }^{1,2+}$, Jun Huang ${ }^{3}$, Yue Zhang ${ }^{3}$, Ying Zhang ${ }^{3}$, Yi Zhang ${ }^{1,2}$, Xiaotian Chen ${ }^{1,2}$, Yun Li ${ }^{3^{*}+}$ and Weili $\operatorname{Yan}^{1,2^{*}+}$ (iD

\begin{abstract}
Background: Early postanal growth of preterm infants has many effects on early and late health. However, evidence on growth pattern in Chinese preterm infant population during early life is insufficient. This study aims to describe the growth trajectory, catch-up growth, and risk of overweight of preterm infants during the first 2 years of life in a Chinese community population.
\end{abstract}

Methods: All preterm infants $(n=10,624)$ received routine childcare in one primary maternal and child healthcare network in 8 years were included. Body weight and length/height at corrected age (CA) 40 weeks, CA 3 months, 6 months, 9 months, 12 months, 18 months, and 24 months were extracted and converted to $z$-scores based on the World Health Organization (WHO) standards. According to the intrauterine growth status, infants were divided into small for gestational age (SGA), appropriate for gestational age (AGA), and large for gestational age (LGA) infants. Changes of z-score were used to describe the growth velocity. Generalized estimating equation (GEE) model was used to analyze growth trajectory trends over time.

Results: Body weight and length/height were overall above the WHO standards during the first 2 years of life. Zscore increased significantly by 0.08 (95\% Cl: $0.06-0.10$ ) in weight and 0.07 (95\% Cl: 0.04-0.09) in length/height from CA 40 weeks to 3 months and then levelled off until CA 24 months after adjustment. Almost 90\% of AGA and LGA infants achieved growth targets ( $\geq 25$ th percentile of WHO standards), and over $85 \%$ of SGA infants achieved catchup growth ( $\geq 10$ th percentile of WHO standards) before CA 24 months. However, the risk of overweight appeared during this period, with the proportion of infants with the risk of overweight being at the peak at CA 3 months (25.6\% of all preterm infants and 39.4\% of LGA infants). Growth trajectories of SGA showed increasing trends, but those of LGA showed decreasing trends during the first 2 years.

(Continued on next page)

\footnotetext{
*Correspondence: liyun32601@226.com; yanwl@fudan.edu.cn

†Junyan Han and Yuan Jiang contributed equally to this work.

${ }^{3}$ Shanghai Minhang District Maternal and Child Health Care Hospital, 805

Gudai Road, Minhang District, Shanghai 201102, China

'Children's Hospital of Fudan University, 399 Wanyuan Road, Minhang

District, Shanghai 201102, China

Full list of author information is available at the end of the article
}

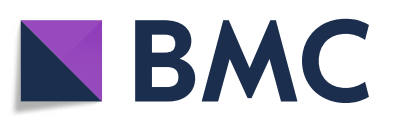

(- The Author(s). 2021 Open Access This article is licensed under a Creative Commons Attribution 4.0 International License, which permits use, sharing, adaptation, distribution and reproduction in any medium or format, as long as you give appropriate credit to the original author(s) and the source, provide a link to the Creative Commons licence, and indicate if changes were made. The images or other third party material in this article are included in the article's Creative Commons licence, unless indicated otherwise in a credit line to the material. If material is not included in the article's Creative Commons licence and your intended use is not permitted by statutory regulation or exceeds the permitted use, you will need to obtain permission directly from the copyright holder. To view a copy of this licence, visit http://creativecommons.org/licenses/by/4.0/. The Creative Commons Public Domain Dedication waiver (http://creativecommons.org/publicdomain/zero/1.0/) applies to the data made available in this article, unless otherwise stated in a credit line to the data. 


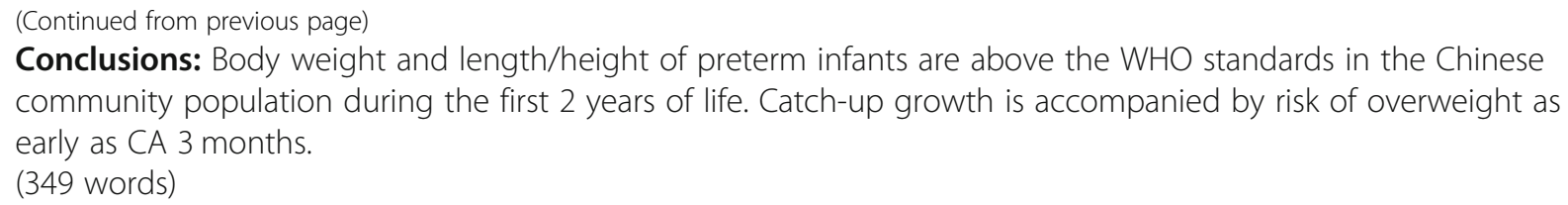

\section{Background}

More than one in 10 of the world's babies born in 2014 were preterm infants, resulting in an estimated 14.8 million preterm births [1]. Because of the vast population base, the number of preterm infants in China ranked second in the world $[1,2]$. Although the survival rate of preterm has increased over past decades along with the development of perinatal and neonatal medicine, they are still more susceptible to growth restriction, delayed motor, and language development in later life [3, 4].

Growth trajectory monitoring is an essential part of children's healthcare, especially for preterm infants. Previous studies on growth trajectory have shown that preterm and/ or low birth weight infants were shorter and lighter than term infants of the same postmenstrual age (PMA). Meanwhile, they often have a rapid growth or catch-up growth period after post-discharge [5, 6]. Early catch-up growth is beneficial for neurodevelopment [7]. Belfort et al.'s study in 945 preterm infants showed rapid growth from term age to 4 months was associated with better intelligence quotient at 18 years old [8]. However, associations between excessive growth in infancy and overweight/obesity, insulin resistance, and elevated blood pressure in childhood or adulthood have been reported in recent studies $[9,10]$. A study in Korean population reported a phenomenon of early accelerated growth or "early catch-up growth" in preterm infants, which might be associated with Asian cultural values and childrearing practices [11]. Nevertheless, evidence on growth pattern or growth trajectory in Chinese preterm infants is still limited.

This study aims to describe the growth trajectory, catch-up growth, and risk of overweight of preterm infants during the first 2 years of life in a communitybased Chinese population, and to compare the growth differences between small for gestational age (SGA) infants, appropriate for gestational age (AGA) infants, and large for gestational age (LGA) infants.

\section{Methods}

\section{Data source and subjects}

Study data were extracted from a routine database of the Maternal and Child Healthcare Network of Minhang district in Shanghai, China. It was a population-based database launched by the government, which recorded all infants' routine healthcare information living in this district constituted of 13 communities for 2.53 million permanent residents. This study included all preterm infants (born before 37 weeks of gestation) who received routine healthcare during the period from January 1, 2010, to December 31, 2017. Infants with a missed or ambiguous birth date, birth weight, or without growth parameter data (body weight and length/height) were excluded. Corrected age (CA) was used in all analyses, defined as the additional age from 40 weeks of PMA. Follow-up data were grouped into CA 40 weeks (40 weeks of PMA), 3 months, 6 months, 9 months, 12 months, 18 months, and 24 months based on their original date of visits. The institutional scientific research department approved this analysis protocol. Personal identification information was removed from the extracted dataset for analyses.

\section{Measurements and definitions}

Body weight and length/height were measured and recorded by trained pediatricians and nurses in community healthcare centers at each routine visit. An electronic scale with $0.01 \mathrm{~kg}$ accuracy was used to measure body weight. Length/height was measured using a length board in a supine position, or a measurement system in standing position reading to the nearest $0.1 \mathrm{~cm}$. For each infant, z-score of measurements were computed using the Lambda-Mu-Sigma (LMS) parameters based on the international standards [12, 13]. The Fenton preterm growth standard (from 22 to 50 weeks of gestation) [12] was used to calculated z-scores at birth, and the World Health Organization (WHO) standards for children $0-5$ years old [13] were used to calculated z-scores from CA 40 weeks to 24 months. According to the intrauterine growth status, all preterm infants were classified as SGA, AGA, and LGA infants, defined as birth weight $<10$ th percentile, 10th-90th percentile, and $>90$ th percentile on the Fenton Growth Chart, respectively [12].

In this study, we defined that growth target was achieved for AGA and LGA infants when the weight or length/height for age $\geq 25$ th percentile of the WHO standards. The growth target for SGA infants was weight or length/height for age $\geq 10$ th percentile of the WHO standards, which meant achieving catch-up growth $[13,14]$. 
Infants whose weight for length $>90$ th percentile of the WHO standard were defined as at risk of overweight $[13,14]$.

\section{Statistical analyses}

Measurement values were included in analyses only if the original date of visits were within 2 weeks around the specific follow-up points. Those measurement values beyond mean \pm three standard deviations (SD) were removed as outliers. Demographics and clinical characteristics were described as mean and SD for continuous variables and absolute numbers with percentages for categorical variables. Growth measurements of our population and the WHO standards were compared using $t$ test for each follow-up time point. Generalized estimating equation (GEE) models were used to analyze trajectory trends of these growth measurements over time. Growth velocity was defined as the change of z-score over the periods $[15,16]$ using the lincom function of GEE models. Gestational age, gender, birth weight zscore, and intrauterine growth status was adjusted as covariates. Stata 16.0 software (Stata Corp, College Station, TX, USA) was used for all the statistical analyses, and the significance level was set at $5 \%$ (two-tailed). Figures were drawn using GraphPad Prism 8.0 for Windows (GraphPad Software, La Jolla California, USA).

\section{Results}

\section{Clinical characteristics}

A total of 10,705 preterm infants met the inclusion criteria. Among them, 80 infants without any follow-up measurement and one infant missing the birth information were excluded. Finally, 10,624 infants were included in the analyses, with gestational age $35.0 \pm 1.8$ weeks (rang $24^{+5}-36^{+6}$ weeks) and birth weight $2463 \pm 520 \mathrm{~g}$. Basic characteristics were shown in Table 1. Among all preterm infants, the majority were late preterm infants (>34 weeks of gestation, 82.7\%) and AGA infants (86.0\%). Over $75 \%$ of infants had highly educated parents (one of the parents received an education in college or above). The body weight $\mathrm{z}$-score was $0.04 \pm 0.91$ at birth.

\section{Growth of preterm infants from CA 40 weeks to CA 24 months}

As shown in Fig. 1 and Additional file 1, body weight increased $7.90 \mathrm{~kg}$ in males and $7.72 \mathrm{~kg}$ in females from CA 40 weeks to CA 24 months. The mean increment in length/height was $33.2 \mathrm{~cm}$ and $33.4 \mathrm{~cm}$ for males and females, respectively. All growth parameters in our study population were significantly above the WHO standards, especially in weight (all $P$-values $<0.001$ ). Growth appears to be faster in male preterm infants than female during the first 2 years of life.
Table 1 Characteristics of all preterm infants [mean \pm SD or $\mathrm{n}(\%)]$

\begin{tabular}{|c|c|}
\hline Characteristics & Total $(n=10,624)$ \\
\hline Male, n (\%) & $5895(55.5)$ \\
\hline Primiparity, n (\%) & $5955(56.1)$ \\
\hline Gestational age, weeks & $35.0 \pm 1.8$ \\
\hline Birth weight, $g$ & $2463 \pm 520$ \\
\hline Birth weight z-score ${ }^{a}$ & $0.04 \pm 0.91$ \\
\hline Caesarean section, n (\%) & $6376(60.0)$ \\
\hline Maternal age, years & $29.7 \pm 4.9$ \\
\hline Paternal age, years & $31.7 \pm 5.9$ \\
\hline$\geq$ college (maternal education) & $6945(76.6)$ \\
\hline$\geq$ college (paternal education) & $7102(78.3)$ \\
\hline \multicolumn{2}{|l|}{ Subgroup by gestational age } \\
\hline$<28$ weeks, n (\%) & $40(0.4)$ \\
\hline $28 \sim 31^{+6}$ weeks, $\mathrm{n}(\%)$ & $642(6.0)$ \\
\hline $32 \sim 33^{+6}$ weeks, n (\%) & $1161(10.9)$ \\
\hline $34 \sim 36^{+6}$ weeks, n (\%) & $8781(82.7)$ \\
\hline \multicolumn{2}{|l|}{ Subgroup by birth weight } \\
\hline$<1000 \mathrm{~g}, \mathrm{n}(\%)$ & $35(0.3)$ \\
\hline 1000-1499 g, n (\%) & $437(4.1)$ \\
\hline $1500 \sim 2499$ g, n (\%) & $4810(45.3)$ \\
\hline$>2500 \mathrm{~g}, \mathrm{n}(\%)$ & $5342(50.3)$ \\
\hline \multicolumn{2}{|l|}{ Intrauterine growth status } \\
\hline SGA, n (\%) & $681(6.4)$ \\
\hline AGA, n (\%) & $9141(86.0)$ \\
\hline LGA, n (\%) & $802(7.6)$ \\
\hline
\end{tabular}

a Z-scores were computed according to the Fenton Preterm Infants Growth Charts;

$S D$ standard deviation; SGA small for gestational age; $A G A$ appropriate for gestational age; $L G A$ large for gestational age

Table 2 showed an estimated change of z-scores (growth velocities) during the six time periods after adjustment. From CA 40 weeks to 3 months, z-score of body weight and length/height increased significantly by 0.08 [95\% confidence interval (CI): 0.06-0.10] and 0.07 (95\%CI: 0.04-0.09), respectively (all $P$-values $<0.001$ ). After that, z-score of body weight declined significantly among periods from CA 3 to 18 months (all $P$-values < 0.001), while with an exception for the change from $C A$ 18 to 24 months $(P=0.071)$. For length/height, the growth velocity also showed a declining trend from $\mathrm{CA}$ 6 to 18 months (all $P$-values $<0.001$ ), while remaining stable from CA 3 to 6 months and from CA 18 to 24 months $(P>0.05)$. Some covariates, including gestational age, birth weight, gender, and intrauterine growth status, showed significant contributions in growth (Additional file 2). 

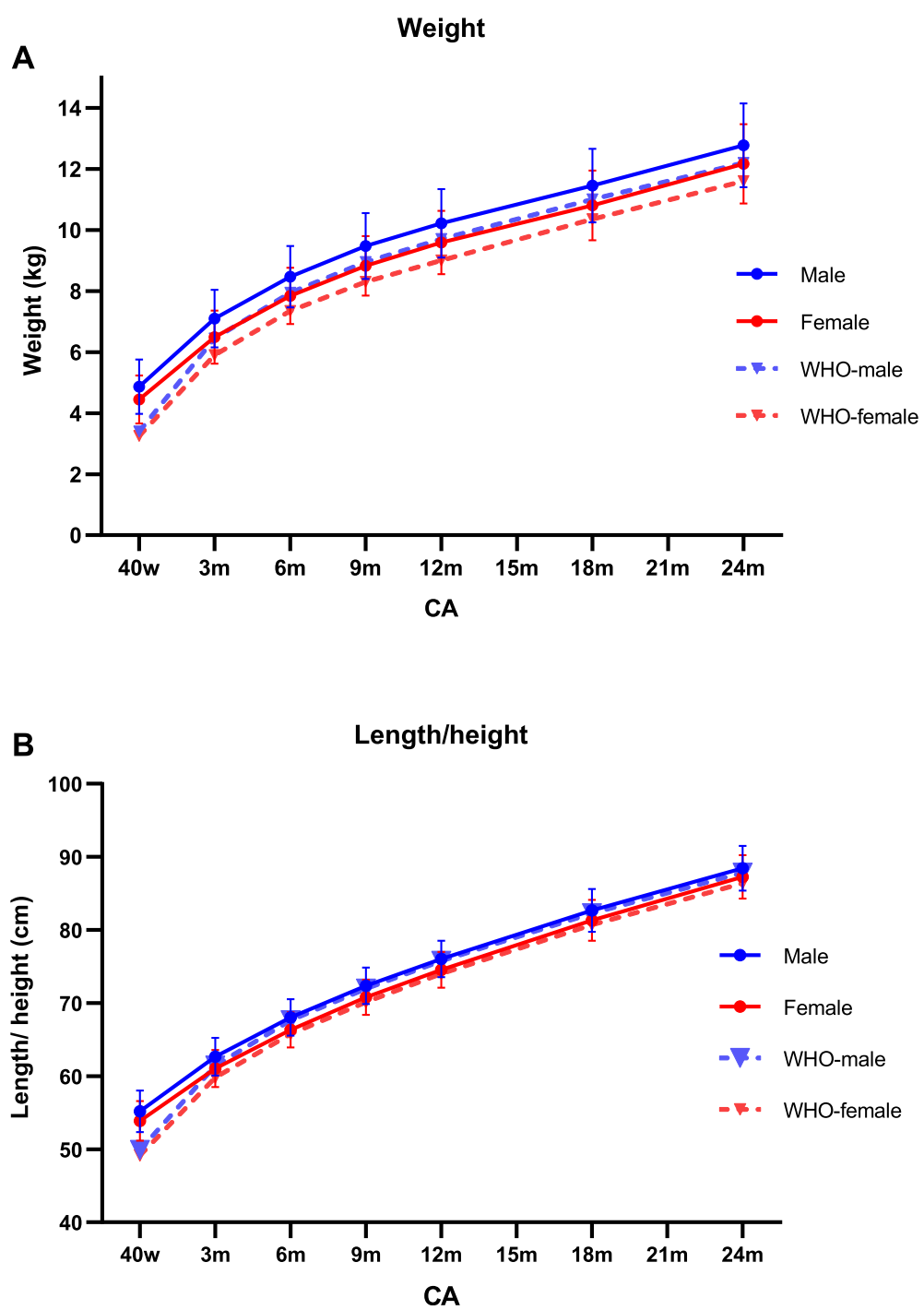

Fig. 1 Growth trajectories of preterm infants from CA 40 weeks to 24 months. a Growth trajectory of body weight in preterm infants and the mean level of the WHO standard. b Growth trajectory of length/height in preterm infants and the mean level of the WHO standards. CA, corrected age; WHO, World Health Organization

Table 2 Change of z-scores in preterm infants from CA 40 weeks to 24 months

\begin{tabular}{|c|c|c|c|c|}
\hline \multirow[t]{2}{*}{ Corrected age } & \multicolumn{2}{|c|}{ Z-score change of weight } & \multicolumn{2}{|c|}{ Z-score change of length/height } \\
\hline & Difference $(95 \% \mathrm{Cl})^{\mathrm{a}}$ & $P$-value & Difference $\left(95 \%\right.$ CI) ${ }^{\mathrm{a}}$ & $P$-value \\
\hline Overall & - & $<0.001$ & - & $<0.001$ \\
\hline CA 40 weeks-3 months & $0.08(0.06-0.10)$ & $<0.001$ & $0.07(0.04-0.09)$ & $<0.001$ \\
\hline CA 3-6 months & $-0.07(-0.09--0.05)$ & $<0.001$ & $0.01(-0.01-0.04)$ & 0.310 \\
\hline CA 6-9 months & $-0.10(-0.12--0.08)$ & $<0.001$ & $-0.07(-0.10--0.05)$ & $<0.001$ \\
\hline CA 9-12 months & $-0.08(-0.10--0.06)$ & $<0.001$ & $-0.09(-0.12--0.07)$ & $<0.001$ \\
\hline CA $12-18$ months & $-0.17(-0.20--0.15)$ & $<0.001$ & $-0.11(-0.14--0.08)$ & $<0.001$ \\
\hline CA $18-24$ months & $-0.02(-0.06-0.00)$ & 0.071 & $0.00(-0.03-0.04)$ & 0.918 \\
\hline
\end{tabular}

${ }^{a}$ Gestational age, gender, birth weight z-score, and intrauterine growth status were adjusted; $C A$ corrected age; $\mathrm{Cl}$ confidence interval 


\section{Achieving growth target and risk of overweight}

At CA 24 months, 92.2 and $89.6 \%$ of AGA infants, and 98.6 and $95.3 \%$ of LGA infants achieved growth target in weight and length/height, respectively (Fig. 2a), and most of them occurred before CA 3 months. Afterward, the proportion of infants achieving growth target kept stable. As for SGA infants, a significant trend of catch-up growth was observed across the 2 years after birth. Especially for the first year of life, the proportion of infants who achieved catch-up growth (reach 10th percentile of the WHO standards) increased from 65.6 to $89.2 \%$ in weight and from 57.2 to $82.8 \%$ in length/height. By the CA 24 months, most SGA infants achieved catch-up growth (90.3\% in weight and $87.3 \%$ in length/height).

The risk of overweight appeared accompanied by catch-up growth (Fig. 2b). At CA 40 weeks, $20.6 \%$ of infants were at risk of overweight. This proportion increased to the peak (25.6\%) at CA 3 months, and then gradually decreased to $14.5 \%$ at CA 24 months. Risk of overweight even occurred in SGA infants, and the proportion ranged from 5.0 to $15.1 \%$ during the first 2 years of life. The proportion of infants with the risk of overweight was significantly higher in LGA infants than that in AGA and SGA infants. Especially at CA 3 months, up to $39.4 \%$ of LGA infants were at risk of overweight.

\section{Growth of SGA, AGA, and LGA preterm infants}

Figure 3 showed z-scores of body weight and length/ height in SGA, AGA, and LGA infants. Differences between these subgroups gradually decreased during the first 2 years of life.

For SGA infants, body weight and length/height zscores were significantly lower than the WHO standards by $0.83 \pm 1.23$ and $1.06 \pm 1.27$ at CA 40 weeks. From CA 40 weeks to CA 24 months, these $\mathrm{z}$-scores showed overall increasing trends, with the mean of $\mathrm{z}$-score increasing by 0.78 in weight and 1.04 in length/height. When CA 24 months, these measurements (body weight $\mathrm{z}$-score $=$
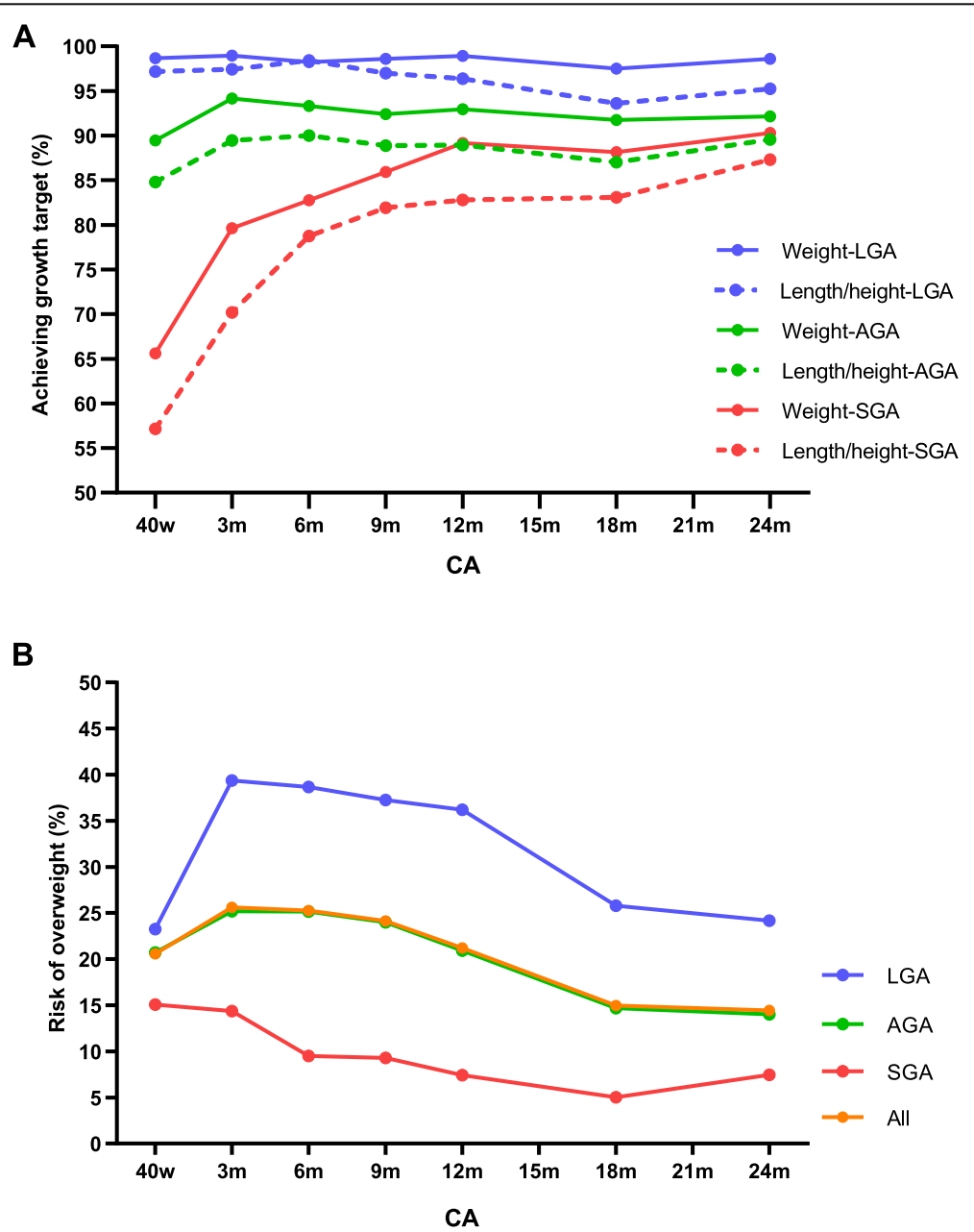

Fig. 2 Achieving target growth and risk of overweight from CA 40 weeks to 24 months in preterm infants. a The proportion of infants who achieved target growth or achieved catch-up growth. $\mathbf{b}$ The proportion of infants with risk of overweight. CA, corrected age; SGA, small for gestational age; AGA, appropriate for gestational age; LGA, large for gestational age 
Weight

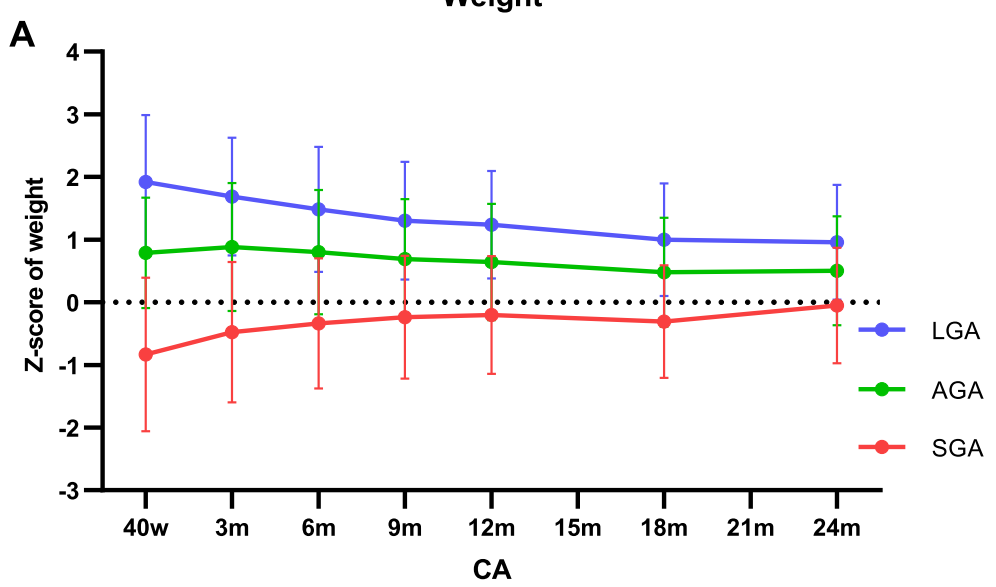

Length/height

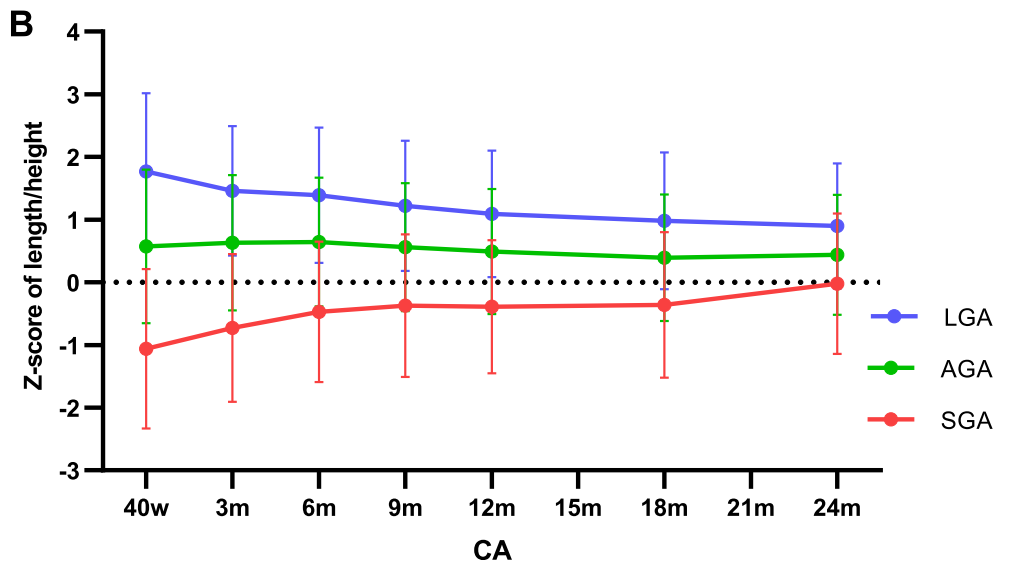

Fig. 3 Growth trajectories of LGA, AGA, and SGA preterm infants from CA 40 weeks to 24 months. a Growth of body weight in LGA, AGA, and SGA infants. $\mathbf{b}$ Growth of length/height in LGA, AGA, and SGA infants. CA, corrected age; SGA, small for gestational age; AGA, appropriate for gestational age; LGA, large for gestational age

-0.05 and length/height $z$-score $=-0.02, \quad$ Additional file 3) were closed to the mean level of WHO standard $(z$-score $=0)$. The period with the fastest growth velocity was from CA 40 weeks to 3 months, weight $\mathrm{z}$-score increased by 0.37 (95\%CI: $0.28-0.45, \mathrm{P}$ value $<0.001)$ and length/height $\mathrm{z}$-score increased by 0.37 (95\%CI: 0.16-0.49, $P$-value < 0.001) (Table 3).

For AGA infants, the means of body weight and length were overall above the WHO standards from CA 40 weeks to 24 months (Fig. 3 and Additional file 3). Zscore increased significantly by 0.08 (95\% CI:0.07-0.11) and 0.07 (95\% CI 0.05-0.10) from CA 40 weeks to 3 months in body weight and length/height, respectively (all $P$-values $<0.001$ ). After that, $\mathrm{z}$-scores of measurements showed overall declining trends until CA 24 months (Table 3).
For LGA infants, z-score of these measurements showed overall declining trends during the first 2 years (Table 3). From CA 40 weeks to 24 months, the mean of z-scores declined by 0.96 in weight and 0.87 in length/ height, respectively (Additional file 3). Z-score of body weight declined significantly from CA 40 weeks to 9 months (all $P$-values $<0.001$ ) and from CA 12 to 18 months (decline by $0.24,95 \% C I=-0.33--0.15, P$-value $<$ 0.001). Length/height $\mathrm{z}$-scores also showed declining trends from CA 40 weeks to 3 months (decline by 0.29 , $95 \% C I=-0.38--0.20, P$-value $<0.001)$ and from CA 6 to 18 months (all $P$-values $<0.001$ ) (Table 3 ).

\section{Discussion}

This study provides evidence on the growth trajectory during the first 2 years of life in a large Chinese preterm 
Table 3 Growth velocities of SGA, AGA, and LGA preterm infants from CA 40 weeks to 24 months

\begin{tabular}{|c|c|c|c|c|c|}
\hline \multirow[t]{2}{*}{ Subgroup } & \multirow[t]{2}{*}{ Corrected age } & \multicolumn{2}{|l|}{ Weight } & \multicolumn{2}{|l|}{ Length/height } \\
\hline & & Difference $(95 \% \mathrm{Cl})^{\mathrm{a}}$ & $P$-value & Difference $(95 \% \mathrm{Cl})^{\mathrm{a}}$ & $P$-value \\
\hline \multirow[t]{7}{*}{ SGA } & Overall & - & $<0.001$ & - & $<0.001$ \\
\hline & CA 40 weeks- 3 months & $0.37(0.28-0.45)$ & $<0.001$ & $0.37(0.16-0.49)$ & $<0.001$ \\
\hline & CA 3-6 months & $0.14(0.06-0.23)$ & 0.001 & $0.27(0.15-0.38)$ & $<0.001$ \\
\hline & CA 6-9 months & $0.05(-0.03-0.15)$ & 0.243 & $0.07(-0.06-0.19)$ & 0.278 \\
\hline & CA 9-12 months & $0.04(-0.06-0.13)$ & 0.433 & $-0.00(-0.13-0.13)$ & 0.992 \\
\hline & CA $12-18$ months & $-0.10(-0.20-0.00)$ & 0.057 & $0.03(-0.10-0.17)$ & 0.640 \\
\hline & CA $18-24$ months & $0.15(0.01-0.29)$ & 0.035 & $0.25(0.07-0.44)$ & 0.008 \\
\hline \multirow[t]{7}{*}{ AGA } & Overall & - & $<0.001$ & - & $<0.001$ \\
\hline & CA 40 weeks- 3 months & $0.08(0.07-0.11)$ & $<0.001$ & $0.07(0.05-0.10)$ & $<0.001$ \\
\hline & CA 3-6 months & $-0.07(-0.09--0.05)$ & $<0.001$ & $0.00(-0.02-0.03)$ & 0.751 \\
\hline & CA 6-9 months & $-0.10(-0.12--0.08)$ & $<0.001$ & $-0.07(-0.10--0.05)$ & $<0.001$ \\
\hline & CA 9-12 months & $-0.09(-0.11--0.07)$ & $<0.001$ & $-0.10(-0.12--0.07)$ & $<0.001$ \\
\hline & CA $12-18$ months & $-0.17(-0.20--0.15)$ & $<0.001$ & $-0.12(-0.14--0.09)$ & $<0.001$ \\
\hline & CA $18-24$ months & $-0.03(-0.06--0.00)$ & 0.032 & $-0.00(-0.04-0.04)$ & 0.999 \\
\hline \multirow[t]{7}{*}{ LGA } & Overall & - & $<0.001$ & - & $<0.001$ \\
\hline & CA 40 weeks- 3 months & $-0.22(-0.29--0.14)$ & $<0.001$ & $-0.29(-0.38--0.20)$ & $<0.001$ \\
\hline & CA 3-6 months & $-0.21(-0.29--0.14)$ & $<0.001$ & $-0.08(-0.17-0.01)$ & 0.069 \\
\hline & CA 6-9 months & $-0.20(-0.29--0.12)$ & $<0.001$ & $-0.18(-0.27--0.08)$ & $<0.001$ \\
\hline & CA 9-12 months & $-0.07(-0.16-0.02)$ & 0.129 & $-0.13(-0.23--0.03)$ & 0.009 \\
\hline & CA $12-18$ months & $-0.24(-0.33--0.15)$ & $<0.001$ & $-0.13(-0.24--0.03)$ & 0.012 \\
\hline & CA $18-24$ months & $-0.03(-0.15-0.09)$ & 0.667 & $-0.08(-0.22-0.05)$ & 0.218 \\
\hline
\end{tabular}

${ }^{a}$ Gestational age, gender, birth weight z-score, and intrauterine growth status were adjusted;

SGA small for gestational age; $A G A$ appropriate for gestational age; $L G A$ large for gestational age; $C A$ corrected age; $C l$ confidence interval

population, showing that the body weight and length/ height were overall higher than the WHO standards. Almost $90 \%$ of AGA infants and over $90 \%$ of LGA infants achieved target growth, and over $85 \%$ of SGA infants achieved catch-up growth before CA 24 months. However, over a quarter of infants had been at risk of overweight as early as CA 3 months, and this proportion reached $39.4 \%$ in LGA infants. To date, we are the first reporting early overweight risk accompanying catch-up growth in the Chinese preterm infant population.

Our study noticed that growth trajectories of preterm infants were consistently over the WHO standards from CA 40 weeks to CA 24 months, especially for weight. Recent studies in Chinese population also found that growth rate of preterm infants was higher than that in term infants during the first year of life [17, 18]. Several possible reasons might explain this phenomenon. Firstly, this study data was from a preterm infant population in community healthcare center, most infants were late preterm infants who were less likely to develop serious diseases and might growth better after birth. Secondly, we believed that rapid growth is directly related to the childrearing practice of their parents, giving their preterm babies more care and feeding to make up the low birth weight. In addition, the "One Child" policy in China before 2016 might be another explanation. Parents and caregivers devote more effort to their only child among these people. A point that the heavier the baby is, the healthier he/she will be is widely accepted [18].

Catch-up growth was important for preterm infants to reduce the risk of stunning and achieve a better neurodevelopment outcome in later life [19-21], especially to SGA infants who had a higher risk of growth retardation and delayed development during infancy and childhood [22, 23]. Olbertz et al.'s study showed that few SGA infants could achieve catch-up growth after age two [24]. Currently, no consensus has reached on which catch-up growth pattern is optimal for preterm infants [25-27]. In our study, most AGA/LGA infants achieved growth targets before the first 3 months. Nevertheless, it occurred lately in other studies, at 6 months or 11-12 months $[28,29]$. The difference in later outcomes between early and later achieving growth target is unclear. Further study is needed to answer this question. In addition, we found that it took more time for SGA preterm infants to achieve catch-up growth. Early aggressive nutrition strategy might be necessary for SGA infants to timely achieve the optimal catch-up growth and healthy 
development, which is in line with a similar point in previous studies $[23,30]$.

One of our main findings was that the growth was accompanied by the risk of overweight in this Chinese preterm infant population. Over a quarter of infants in this population are at risk of overweight as early as CA 3 months, and the overweight risk is even higher in LGA preterm infants. Excessive weight gains during early life in preterm infants were linked with a higher prevalence of type 2 diabetes and cardiovascular diseases in adulthood [31-34]. Kerkhof et al. observed that gain in weight for length of preterm infants during the first 3 months after term age was associated with greater body fat percentage, waist, serum cholesterol, and low-density lipoprotein in early adulthood [35]. Therefore, given these reports of adverse effects on adult health, controlling the risk of overweight during the same period of promoting optimal catch-up growth in preterm infants is becoming a challenge in primary child healthcare practice [3, 21]. Different risk-balance considerations in different preterm infants are needed, and one size might not fit all [36].

\section{Strengths and limitations}

Based on a local community-based child healthcare network, this study has the largest sample size of Chinese preterm infant population on growth trajectory of preterm infants compared with published reports before, while some limitations are inevitable. Firstly, the study sample consists of a greater proportion of late preterm infants $(82.7 \%)$, higher than that in a published nationwide survey [37]. This may be partly explained by the fact that very preterm infants or very low birth weight infants are more likely to be admitted in the neonatal intensive care unit (NICU) of tertiary hospitals and followed up at there, rather than at community healthcare centers. Besides, the proportion of late preterm infants becomes greater $(2562 / 2824,90.7 \%)$ at CA 24 months. Thus, the estimations of growth status and trends in our study may be overestimated and more generalizable for late preterm infants or preterm infants born relatively healthy. In addition, data of maternal diseases history, neonatal diseases, and feeding information are not available in our study, their influences on the status and trends of growth are not investigated and warrant further prospective well-designed studies.

\section{Conclusions}

Body weight and length/height of preterm infants in the Chinese community population are above the WHO standards during the first 2 years of life, and catch-up growth is accompanied by risk of overweight, which occurred as early as CA 3 months. Although the optimal growth pattern is not fully established, more attention is needed to promote the proper catch-up growth of preterm infants for better long-term outcomes.

\section{Abbreviations}

AGA: Appropriate for gestational age; CA: Corrected age; Cl: Confidence interval; GEE: Generalized estimating equation; LGA: Large for gestational age; LMS: Lambda-Mu-Sigma; NICU: Neonatal intensive care unit; PMA: Postmenstrual age; SD: Standard deviation; SGA: Small for gestational age infants; WHO: World Health Organization

\section{Supplementary Information}

The online version contains supplementary material available at https://doi. org/10.1186/s13052-021-01019-2.

Additional file 1. Growth of preterm infants from CA 40 weeks to 24 months compared with the WHO standards (mean \pm SD).

Additional file 2. Covariates associated with growth velocity of preterm infants.

Additional file 3. Body weight and length/height z-scores of SGA, AGA, and LGA infants from CA 40 weeks to 24 months (mean \pm SD).

\section{Acknowledgements}

The authors thank all the nurses and technicians of Maternal and Child Health Care Network in Minhang District for collecting data who were not included in the author list.

Authors' contributions

$W Y$ and $Y L$ conceived the study and guided the data analysis. JH and $Y J$ drafted the manuscript and analyzed the data. YiZ and XC contributed to analyzing data and making charts. JuH, YuZ, and YingZ collected and controlled the quality of data. All authors were involved in writing the paper, interpreting the results, and had final approval of the submitted and published versions.

\section{Funding}

This work was financially supported by the National Natural Science Foundation of China (Grant No. 81273168).

Availability of data and materials

The datasets used and/or analyzed during the current study are available from the corresponding author on reasonable request.

\section{Declarations}

Ethics approval and consent to participate

The institutional scientific research department approved the analysis protocol.

Consent for publication

Not applicable.

\section{Competing interests}

The authors declare that they have no competing interests.

\section{Author details}

${ }^{1}$ Children's Hospital of Fudan University, 399 Wanyuan Road, Minhang District, Shanghai 201102, China. 'National Children's Medical Center, 399 Wanyuan Road, Minhang District, Shanghai 201102, China. ${ }^{3}$ Shanghai Minhang District Maternal and Child Health Care Hospital, 805 Gudai Road, Minhang District, Shanghai 201102, China.

Received: 11 July 2020 Accepted: 2 March 2021

Published online: 16 March 2021

\section{References}

1. Chawanpaiboon S, Vogel JP, Moller AB, Lumbiganon P, Petzold M, Hogan D, et al. Global, regional, and national estimates of levels of preterm birth in 
2014: a systematic review and modelling analysis. Lancet Glob Health. 2019; 7(1):e37-46.

2. Howson CP, Kinney MV, McDougall L, Lawn JE. Born too soon: preterm birth matters. Reprod Health. 2013;10(Suppl 1):S1.

3. Streimish IG, Ehrenkranz RA, Allred EN, O'Shea TM, Kuban KC, Paneth N, et al. Birth weight- and fetal weight-growth restriction: impact on neurodevelopment. Early Hum Dev. 2012;88(9):765-71.

4. Dusick AM, Poindexter BB, Ehrenkranz RA, Lemons JA. Growth failure in the preterm infant: can we catch up? Semin Perinatol. 2003;27(4):302-10.

5. Roberts G, Cheong J, Opie G, Carse E, Davis N, Duff J, et al. Growth of extremely preterm survivors from birth to 18 years of age compared with term controls. Pediatrics. 2013;131(2):e439-45.

6. Bocca-Tjeertes IF, van Buuren S, Bos AF, Kerstjens JM, Ten Vergert EM, Reijneveld SA. Growth of preterm and full-term children aged 0-4 years: integrating median growth and variability in growth charts. J Pediatr. 2012; 161(3):460-5 e1.

7. Bocca-Tjeertes IF, Kerstjens JM, Reijneveld SA, de Winter AF, Bos AF. Growth and predictors of growth restraint in moderately preterm children aged 0 to 4 years. Pediatrics. 2011;128(5):e1187-94.

8. Belfort MB, Gillman MW, Buka SL, Casey PH, McCormick MC. Preterm infant linear growth and adiposity gain: trade-offs for later weight status and intelligence quotient. J Pediatr. 2013;163(6):1564-9 e2.

9. Singhal A. Should we promote catch-up growth or growth acceleration in low-Birthweight infants? Nestle Nutr Inst Workshop Ser. 2015;81:51-60.

10. Ekelund $U$, Ong KK, Linne $Y$, Neovius M, Brage S, Dunger DB, et al. Association of weight gain in infancy and early childhood with metabolic risk in young adults. J Clin Endocrinol Metab. 2007;92(1):98-103.

11. Ahn $Y$, Sohn $M$, Jun $Y$, Lee $S$. Growth patterns and their implications for preterm infants in a culture of rapid modernization. J Child Health Care. 2013;17(3):242-52

12. Fenton TR, Nasser R, Eliasziw M, Kim JH, Bilan D, Sauve R. Validating the weight gain of preterm infants between the reference growth curve of the fetus and the term infant. BMC Pediatr. 2013;13:92.

13. WHO child growth standards: length/height-for-age, weight-for-age, weight-for-length, weight-for-height and body mass index-for-age: methods and development. Geneva: World Health Organization; 2006. www.who.int/ childgrowth/standards/en/.

14. Editorial board of the Chinese journal of pediatrics, The Subspecialty Groups of Child Health Care, The Society of Pediatrics, Chinese Medical Association Neonatology group, The Society of Pediatrics, Chinese Medical Association. Post-discharge feeding recommendations for preterm or low-birth-weight infants. Chin J Pediatr. 2016;54(1):6-12.

15. Fenton TR, Chan HT, Madhu A, Griffin IJ, Hoyos A, Ziegler EE, et al. Preterm Infant Growth Velocity Calculations: A Systematic Review. Pediatrics. 2017; 139(3):e20162045.

16. Simon L, Hanf M, Frondas-Chauty A, Darmaun D, Rouger V, Gascoin G, et al. Neonatal growth velocity of preterm infants: the weight Z-score change versus Patel exponential model. PLoS One. 2019;14(6):e0218746.

17. Zhang L, Li Y, Liang S, Liu XJ, Kang FL, Li GM. Postnatal length and weight growth velocities according to Fenton reference and their associated perinatal factors in healthy late preterm infants during birth to termcorrected age: an observational study. Ital J Pediatr. 2019;45(1):1.

18. Kang L, Wang H, He C, Wang K, Miao L, Li Q, et al. Postnatal growth in preterm infants during the first year of life: a population-based cohort study in China. PLoS One. 2019;14(4):e0213762.

19. Cooke RJ. Postnatal growth and development in the preterm and small for gestational age infant. Nestle Nutr Workshop Ser Pediatr Program. 2010;65: 85-95 discussion 6-8.

20. Raaijmakers A, Allegaert K. Catch-Up Growth in Former Preterm Neonates: No Time to Waste. Nutrients. 2016;8(12):817.

21. Victora CG, Barros FC, Horta BL, Martorell R. Short-term benefits of catch-up growth for small-for-gestational-age infants. Int J Epidemiol. 2001;30(6): 1325-30.

22. Clayton PE, Cianfarani S, Czernichow P, Johannsson G, Rapaport R, Rogol A. Management of the child born small for gestational age through to adulthood: a consensus statement of the international societies of pediatric endocrinology and the growth hormone research society. J Clin Endocrinol Metab. 2007;92(3):804-10.

23. Kesavan K, Devaskar SU. Intrauterine growth restriction: postnatal monitoring and outcomes. Pediatr Clin N Am. 2019;66(2):403-23.
24. Olbertz DM, Mumm R, Wittwer-Backofen U, Fricke-Otto S, Pyper A, Otte J, et al. Identification of growth patterns of preterm and small-for-gestational age children from birth to 4 years - do they catch up? J Perinat Med. 2019; 47(4):448-54.

25. Villar J, Giuliani F, Barros F, Roggero P, Coronado Zarco IA, Rego MAS, et al. Monitoring the Postnatal Growth of Preterm Infants: A Paradigm Change. Pediatrics. 2018;141(2):e20172467.

26. Singhal A, Fewtrell M, Cole TJ, Lucas A. Low nutrient intake and early growth for later insulin resistance in adolescents born preterm. Lancet. 2003;361(9363):1089-97.

27. Fanaro $\mathrm{S}$. Which is the ideal target for preterm growth? Minerva Pediatr. 2010;62(3 Suppl 1):77-82.

28. Zhao R, Xu L, Wu ML, Huang SH, Cao XJ. Maternal pre-pregnancy body mass index, gestational weight gain influence birth weight. Women Birth. 2018;31(1):e20-e5.

29. Gong YH, Ji CY, Shan JP. A longitudinal study on the catch-up growth of preterm and term infants of low, appropriate, and high birth weight. Asia Pac J Public Health. 2015;27(2):NP1421-31.

30. Morag I, Stern Levkovitz O, Siman-Tov M, Frisch M, Pinhas-Hamiel O, Strauss T. Postnatal Growth Disadvantage of the Small for Gestational Age Preterm Twins. Nutrients. 2018;10(4):476.

31. Stettler N, Zemel BS, Kumanyika S, Stallings VA. Infant weight gain and childhood overweight status in a multicenter, cohort study. Pediatrics. 2002; 109(2):194-9.

32. Stettler N, Kumanyika SK, Katz SH, Zemel BS, Stallings VA. Rapid weight gain during infancy and obesity in young adulthood in a cohort of African Americans. Am J Clin Nutr. 2003;77(6):1374-8.

33. Embleton ND, Korada M, Wood CL, Pearce MS, Swamy R, Cheetham TD. Catch-up growth and metabolic outcomes in adolescents born preterm. Arch Dis Child. 2016;101(11):1026-31.

34. Cameron N, Pettifor J, De Wet T, Norris S. The relationship of rapid weight gain in infancy to obesity and skeletal maturity in childhood. Obes Res. 2003;11(3):457-60.

35. Kerkhof GF, Willemsen RH, Leunissen RW, Breukhoven PE, Hokken-Koelega AC. Health profile of young adults born preterm: negative effects of rapid weight gain in early life. J Clin Endocrinol Metab. 2012;97(12):4498-506.

36. Singhal A. Long-term adverse effects of early growth acceleration or catchup growth. Ann Nutr Metab. 2017;70(3):236-40.

37. Chen C, Zhang JW, Xia HW, Zhang HX, Betran AP, Zhang L, et al. Preterm birth in China between 2015 and 2016. Am J Public Health. 2019;109(11): 1597-604.

\section{Publisher's Note}

Springer Nature remains neutral with regard to jurisdictional claims in published maps and institutional affiliations.

Ready to submit your research? Choose BMC and benefit from:

- fast, convenient online submission

- thorough peer review by experienced researchers in your field

- rapid publication on acceptance

- support for research data, including large and complex data types

- gold Open Access which fosters wider collaboration and increased citations

- maximum visibility for your research: over $100 \mathrm{M}$ website views per year

At BMC, research is always in progress.

Learn more biomedcentral.com/submissions 